\title{
Innovative Basis of Historical and Ethnographic Research of Art Pottery of Ancient Ganja
}

\author{
Ph.D. Həsənov Elnur Lətif
}

\author{
Ganja Branch of ANAS, Ganja, Azerbaijan; I-hasan@hotmail.com
}

\section{Doi:10.5901/mjss.2016.v7n1s1p17}

\begin{abstract}
In this scientific paper for the first time have been systematic researched the basic features of development of art pottery in ancient Ganja on the basis of different historic sources and scientific materials. Also was investigated the importance of these local pottery wares.
\end{abstract}

Keywords: pottery, ethnography, Azerbaijan, Ganja, ancient national handicraft traditions

\section{Introduction}

Ganja, città natale del grande poeta e pensatore Nizami Ganjavi è una delle città più antiche d'Oriente. Come sapete, dal momento che il VII-VI millennio $\mathrm{aC}$ in Ganja sviluppato ceramica. Che è già nel Neolitico, Calcolitico, il territorio dell'antica Ganja, così come la Valle Ganjachay producono ceramiche presto. Grazie al miglioramento di tradizioni artigianali, con i campioni di tempo di ceramica sono diventati più diversificati e di qualità [Hasanov, E.L. 2010a].Va notato che nella storia della ceramica d'arte fine Ganja dell'età del ferro e il primi secoli dC, sono di grande importanza. In questo epoca antica vi è un elevato miglioramento delle forme strutturali e di plastica, così come le caratteristiche decorative e ornamentali di ceramiche artistiche dell'antico Stato dei nostri antenati - l'Albania caucasica.

Ganja è un importante centro culturale ed economico dello Stato. II principale valore storico ed etnografico dei prodotti di ceramica artistica Ganja è il fatto che la ceramica è stata una visione di primo piano e decisivo di influenzare il resto delle arti e mestieri di Albania caucasica nel periodo in esame. VI secolo aC I secolo aC è considerato il periodo di massimo splendore di ceramiche artistiche dell'antica Ganja. In questa epoca possono essere rintracciate forme ceramiche tradizionali la continuazione di precedenti periodi storici. Oltre a continuare ad agire e antichi rituali religiosi dell'età del bronzo e prima età del ferro, spesso influenzare la creazione di forme zoomorfe in ceramica tradizionali come vasi comunicanti e altri. Inoltre, in questo periodo storico, mentre ci sono anche i vasi che si basano sulle esigenze di vita familiare. Esempi includono vasi, barattoli e altre navi allo sviluppo di forme ceramiche [Hasanov, E.L. 2010b; Almamedov, H.I. 2007]. E 'necessario prendere in considerazione l'impatto diretto della struttura economica di Albania caucasica nello sviluppo della produzione ceramica in grandi città come Ganja. Così, l'organizzazione del settore ceramico in molti modi è stato associate con lo sviluppo dell'economia nazionale. Un esempio è il fatto che il trasporto di argilla, consegna dei materiali da costruzione per la costruzione di forni, la disponibilità di legna da ardere, capacità consumatore della popolazione in ceramica e altri importanti processi sono stati stimolati condizione economica della società antica. Ma a differenza dei vasi di culto, valore brocche domestiche appare immediatamente sulla base della struttura economica del Paese. Vasi, vasetti e altri natanti simili sono un esempio.

La caratterizzazione scientifica storico ed etnografico di ceramiche artistiche dell'antica Ganja questo periodo di grande importanza è lo sviluppo di forme costruttive di plastica e le caratteristiche della ceramica. Le principali forme strutturali e plastiche di ceramiche artistiche dell'antica periodo Albania caucasica sono:

1. Vasi di ceramica. 2. Vaso artistico. 3. Ceramica forma. 4. Vasi comunicanti. 5. Yaloylutepe tipi di navi.

\section{Literature Review}

Sul territorio del vecchio Ganja, così come alcuni siti storici e archeologici della valle del fiume Ganjachay è stato trovato un bel po 'di dati di esempio i principali gruppi di prodotti ceramici [Sulimirski, T.S. 1954]. Vasi di ceramica come una delle principali forme strutturali e plastiche di ceramiche artistiche dell'antica Ganja determinato periodo storico, non a caso rivivere in ceramica, come sono stati strettamente legati alla vita economica dei nostri antenati e caratteristiche geografiche.

Questi prodotti ceramici sono alcune delle caratteristiche e in base alla composizione specifica della struttura 
possono essere suddivisi in tre gruppi tipologici: a) II primo gruppo comprende vasi, caratterizzati dalla forma semisferica del corpo, il collo, e ampiamente utilizzato due grandi maniglie laterali. b) il secondo gruppo sono caratterizzati dal vaso con due manici sulle spalle e una specie di lato convesso. Questi includono flaconi senza becco e con beccuccio che sono progettati per essere indossati nel sacchetto in posizione verticale. c) un terzo gruppo comprende vaso di ceramica tipologica, le sue caratteristiche compositive differenti dai modelli precedenti. Poiché le navi in questo gruppo sulla base genetica e la struttura composita di diverse forme di direzioni orizzontale e verticale del coperchio della custodia. È importante sottolineare che le caratteristiche artistiche e plastiche di ceramiche dell'antica Ganja particolarmente forte nei vasi curve. Essi sono anche parte integrante dell'arte plastica del Albania caucasica.

Rappresentazioni religiose e riti religiosi hanno contribuito alla creazione di vasi curve che passano di generazione in generazione, sono stati la ragione principale per la continuazione di questo tipo di ceramica artistica tradizionale. A questo proposito, è da notare che le diverse forme d'arte a forma di vasi dell'età del bronzo, e ripetute in epoche successive - nella prima età del Ferro nei secoli IV-I aC, e anche nei primi Medioevo. I principali vasi figurativi di questo periodo sono le ceramiche zoomorfe. Sul territorio di Iran, Turchia (Asia Minore), I'Asia centrale sono stati scoperti esemplari di ceramiche, vasi che ricordano l'antica periodo di Ganja [Dyson, R. 1960; Herzfeld, E. 1941].

\section{Research Methodology}

Antropomorfi, zoomorfi e motivi geometrici d'arte ceramiche dell'antica Ganja e zoomorfa (ricci) le navi, hanno molte somiglianze con modelli di ceramica di siti storici e archeologici come Bampur, Tepe Giyan, Persepolis, Shahi Tumpen, Giya e altri. Questo nuovo Dimostra l'importanza della ceramica in Ganja e tutta la regione come un Gyandzhabasarsk fonti etnografiche nello studio del passato, non solo in Azerbaijian, nel Caucaso, ma anche l'intera area Vicino e Medio Oriente. Questi

campioni di ceramiche artistiche dell'antica Ganja è un indicatore degli antichi rapporti etnico culturale nella regione durante il Bronzo, prime età del ferro, così come nel periodo ellenistico [Young, C. 1965; Stagul, G. 1969]. Inoltre, una varietà di forme artistiche di vasi a forma di plastica possono diffondere le loro cinque gruppi tipologici:

1) II primo gruppo tipologica sono le navi zoomorfi, che gli antichi maestri Ganja ha dato la forma di animali o di uccelli diversi. Questi vasi sono stati utilizzati nella commissione dei rituali, così come per l'uso domestico, come i vasi dell'Acquario. Questi vasi zoomorfi realizzate a forma di anatre, piccioni, oche, mucche, cervi, capre e altri animali, e con l'apertura della bocca per versare liquidi, varie ed interessanti per la loro costruttiva, plastica e stilemi;

2) Il secondo gruppo di navi zoomorfa tipologia del periodo sono le navi che si trovano su maniglie e beccucci, nonché sotto il naso e intorno a loro sono modellati in un stilizzate immagini di animali, una forma abbreviata. Campioni di questo gruppo dalla natura delle immagini stucco non sono identiche, sono originali caratteristiche distintive [Ismizade, O.Sh. 1956].

3) II terzo gruppo, che porta le caratteristiche di entrambe le forme d'arte vecchi e nuovi, è un misto e complesso. La caratteristica principale è che il corpo del vaso dall'esterno è decorato con un rilievo raffigurante animali in tutto 0 in forma abbreviata: 4) II quarto gruppo tipologico comprende recipienti di ceramica, che sono attaccati alle figure e teste mezzo-animale di plastica. Una caratteristica specifica dei campioni di questo gruppo sta nel fatto che durante la separazione dei semi-figure e il prodotto teste dai vasi rimangono forma artistica ceramica, anche se essi sono combinati nella composizione totale. La caratteristica principale di questo gruppo è che i dati non sono generalmente vicino al bordo del recipiente e sono collegati direttamente agli alloggiamenti di lanciatori. Tutti rhytons con figure di animali appartengono a questo gruppo; 5) II quinto gruppo di navi zoomorfi di questo periodo storico sono ceramiche con manopole, le figure animali Corolla e prugne che compongono la parte finale delle composizioni di plastica [Hasanov, E.L. 2010b; Almamedov, H.I. 2007]. Come sapete, l'unica forma di ceramiche artistiche di antica Albania, così come Ganja è vaso artistico. Queste ceramiche a causa delle sue utilitaristici vasi considerati. Vaso artistico si trova sul territorio di Ganja e tutto regione Gyandzhabasar hanno un sacco di caratteristiche uniche.

Ci sono vasi con alloggiamenti emisferica, a tre gambe, con numerose varianti. Tale forma arcaica di vasi può essere sorto sulla base di bisogni religiosi. Questi tipi di vasi sono ampiamente diffuse in tutto il Caucaso ed i paesi del Medio Oriente. In alcuni vasi artistico spalla forma sferica dello scafo è decorato con dossi ritmicamente ripetitive, che sono fatti dalla pressione interna del dito. Corolle vasi stanno bordo circolare che viene sollevato sopra il corpo di forma sferica, e solo in un posto si trasforma in una, rilassato forma ondulata [Museibli, N.A. 2004]. 


\section{Conclusion and Recommendations}

Scientific and arch Inoltre, i tipici vasi in ceramica artistica dell'antico Ganja sono prodotti (vasi) con ruote organo che ha tre gambe 0 un vassoio sottile che consiste in una forma cilindrica e la base circolare. Questi prodotti acquisiscono valore artistico grazie alla bellezza plastica e l'originalità della sua composizione di forme ceramiche. Questi vasi forma di gambe possono essere una vita divisa in due gruppi: II primo gruppo - il prodotto con tre gambe; II secondo gruppo - un vaso di pallet [Fomenko, V.P. 1957]. Vaso Artistico antica Ganja, nato sulla base delle esigenze religiose, naturalmente, si tratta di un grande risultato creativo dei nostri antenati, in particolare ceramite, e uno dei segni più chiari della cultura materiale del Albania caucasica. Vasi di questo periodo storico, la popolazione utilizzato per decorare le loro condizioni di vita. Si può concludere sulle alte esigenze culturali e sociali dei nostri antenati. Uno dei prodotti più importanti di questo periodo storico di antica Ganja e l'intera Albania caucasica sono vasi comunicanti. Questi campioni in ceramica sono accoppiati progettazione. Per questo motivo questo gruppo di campioni di ceramica sono caratterizzati da caratteristiche più complesse arte composizione [Golubkina, T.I. 1949]. La maggior parte dei vasi comunicanti, questo periodo storico sono stati trovati sul territorio della regione Gyandzhabasarsk nella Ganjachay valle. Ci sono suggerimenti che i vasi zoomorfi e dei vasi erano strettamente collegati nei tempi antichi. In questo contesto, si può vedere scopo ritual vasi comunicanti.

Alcuni esempi di vasi comunicanti antica Ganja rappresentata da tre fiala senza maniglie rosa pallido, che collega il tronco in una singola composizione. Le navi si trovano in spalla Mingachevir, tradizionalmente ornate incandescente in due file, che hanno quasi nessuna influenza sul set di ceramica artistica. Va notato che l'arte in questo caso non si ottiene a spese della decorazione, è causa di un semplice forme costruttive di navi e loro composti architettonica [Hasanov, E.L. 2010b; Almamedov, H.I. 2007].

Altri esempi di vasi comunicanti che sono vasi di ceramica grigio-argilla, si differenziano per le loro caratteristiche strutturali e decorativi. Maniglie di navi, nonché le ulteriori ponti forma ceramica tra i tre dipendenti posta navi, pesanti con monolite ceramico. Yaloylutepe vasi tipi sono stati rilevati in Yaloylutepe, per questo motivo, i cosiddetti [Museibli, N.A. 2004]. Questi prodotti ceramici artistici e diverse composizioni di plastica sono valore artistico e ornamentale. Poiché questi vasi sono caratterizzati da lungo becco e prugne profonde, forma sfera-biconica dello scafo, maniglie laterali e argilla giallo-rosa. Va notato che il periodo storico analizzato è l'era dello sviluppo di colture come sepolture di cultura con un po 'accovacciato scheletro, la cultura e yaloylutepe vaso-sepoltura. L'era della cultura associata a secoli yaloylutepinsk II. BC. L'importante spettacolo storico ed etnografico di prodotti di ceramica artistica dell'antica Ganja questo periodo sono elementi decorativi e ornamentali. In primo luogo, il particolare valore artistico di stampi ceramici hanno elementi strutturali. II corpo, il collo e punta frusta e penna sono importanti fonti di studio di antiche ceramiche Ganja. Inoltre, i tipi di decorazione artistica di recipienti di ceramica sono considerate indicatori tangibili. I principali elementi decorativi e ornamentali della antica arte della ceramica e l'intero azero Ganja data epoca sono ornamenti di colore, mobili rilievo-decorativi, anche in modo grafico la decorazione. Va notato che il bianco incrostato ceramiche dell'antica Ganja, in alcuni casi, si applicano non solo ai Bronzo finale e prima Periodo di ferro, ma anche a questa epoca antica [Hasanov, E.L. 2010a; Hasanov, E.L. 2009]. Articoli di ceramiche artistiche dell'antica periodo antico Ganja, senza dubbio è una fonte molto importante ricerca storica e etnografica come il passato storico di Azerbaijian e tutto il Medio Oriente. A questo proposito, elementi decorativi e ornamentali di campioni ceramici di Ganja sono di particolare importanza. Recipienti di ceramica incisa antica Ganja spesso caratterizzate dall'uso di massa bianca e motivi ornamentali quali cerchi, triangoli, e altri. Queste decorazioni si trovano principalmente nella ceramica grigio-argilla IV-III sec. BC. Alcuni recipienti di ceramica sono stati rilevati segno. Questo è un segno precoce dell'uso di ceramiche in Albania caucasica timbro con la forma standard del logo. La loro tipo e dimensione della sporgenza centrale globale suggerisce che essi sono stati depositati sul vaso viene estrusa e la mano. Va notato che nel Medioevo e lo stigma è stato applicato a mano e punzonatura [Vaidov, R.M. (1961).] e aveva molte varietà di modello [Kuftin, B.A. 1976]. Inoltre, uno dei più importanti e gli elementi più suggestivi del rilievodecorazione di Albania caucasica, così come Ganja, una decorazione vasi corpo scanalate. Questa tecnica è ancora utilizzato da ceramisti di Ganja in VII-V secolo aC. Questi flauti, dividendo il corpo in larga parte sporgente in verticale, danno il vaso decorativo originale e l'armonia. Chiaramente intagliato flauto accompagnati da un collo alto e combinato con la forma contorta del manico. Motivi decorativi distrarre l'attenzione dalla nave stampaggio ruvida e arricchiscono le sue probabilità di composizione plastica. Essi non distorcono la forma strutturale dello scafo e non lasciano in ombra, al contrario, sottolineano i suoi vantaggi nella composizione complessiva della nave e la nave rende più interessante. Processo fancy dipinto è caratterizzato da loro motivi e le tecniche di prestazioni decorative. Questo metodo di decorazione di vasi di ceramica presenti nel prodotto tinto di Albania caucasica, tra cui Ganja e Ganjabasar [Eminli, J.T. 2008; Gasanov, E.L. 2014a]. I campioni di ceramica artistica Ganja determinato periodo storico sono una fonte significativa di imparare l'epoca ellenistica e la storia antica di Azerbaijian. A 
causa di studio storico e etnografico ha rivelato molte caratteristiche, come pure la genesi della tradizione artigianale di ceramiche antiche Ganja.

\section{References}

Hasanov, E.L. (2010a). Samples of pottery of ancient Ganja early Iron Age as a source of ethnographic research in general for Azerbaijan and Georgia pottery. Kutaisi Scientific Centre. Magazine "Innovation"., 131-135.

Hasanov, E.L. (2010b). To a question about the development of pottery. "Actual problems of the history of Ganja" II Scientific Conference (materials)., p. 25-30.

Almamedov, H.I. (2007). Painted Pottery settlements Polulete. Latest archaeological and ethnographic studies in the Caucasus. International Scientific Conference "Archaeology, Ethnology and Folklore of the Caucasus.", 72-73.

Hasanov, E.L. (2009). About the period of primary formation of the ancient Ganja ceramics trade and its historic-ethnographical description. Journal "Scientific News". Ganja Regional Scientific Centre of Azerbaijan NAS., 124-126.

Sulimirski, T.S. (1954). Scythian Antiquities in Western Asia. (2nd ed.). Calcutta: Science. V. 17.

Dyson, R. (1960). Early Cultures in Azerbaijan, Iran. Proceeding of the Fourth International Congress of Persian Art and Archeology., p.56.

Herzfeld, E. (1941). Iran in the Ancient East. (3rd ed.). London: Cambridge.

Young, C. (1965). Comparative of ceramic chronology for Western Iran. V. 3.

Stagul, G. (1969). Excavations near Ghaligai and Chronogical Sequence of Proto-historical Cultures in the Swat Valley. "East and West". V.19.

Stein, A. (1931). An Archaeological Tour in Gedrosia. Memoires of the Archaeological Survey of India.

Trever, K.V. (1959). Essays on the history and culture of Caucasian Albania, 323-324.

Ismizade, O.Sh. (1956). Yaloylutepe culture. Baku: Ganjlik.

Kaziev, S.M. (1960). Album pitcher burials of Mingechevir. Baku: Elm.

Museibli, N.A. (2004). Archaeological excavations in the necropolis Zeyamchaysk. Archaeology and Ethnography of Azerbaijan.

Fomenko, V.P. (1957). "Connecting" vessels of Mingechevir. Proceedings of the Azerbaijan Museum of History. V. 2.

Golubkina, T.I. (1949). Stamps on Mingechaur ceramics. Reports of the Azerbaijan.

Vaidov, R.M. (1961). Mingachevir in III-VIII centuries. Baku: Elm.

Kuftin, B.A. (1976). Archaeological excavations in the Trialeti. Tbilisi: Science.

Eminli, J.T. (2008). Painted pottery from the Hellenistic period Azerbaijan. "Proceedings" of the National Academy of Sciences of Azerbaijan, 49-59.

Gasanov, E.L. (2014a). O razvitii traditsionnykh remeslennykh otrasley Gyandzhi na rubezhe XIX-XX vekov. Fundamental'nye issledovaniya. 9(4), 892-895.

Gasanov, E.L. (2014b). Traditsionnye etnoantropologicheskie i istoricheskie osobennosti osnovnykh remeslennykh otrasley Gyandzhi XIX - nachala XX v. Vestnik Leningradskogo gosudarstvennogo universiteta im. A.S. Pushkina. T. 4(3)., 86-90.

Nishiaki, Y., \& Hasanov, E.L. (2014). About ethnoarchaeological and anthropological research of some prehistoric monuments of Ganja. ISJ Theoretical \& Applied Science, 1, 45-48. doi: http://dx.doi.org/10.15863/TAS.2014.01.9.8

Smith, W.B., \& Hasanov, E.L. (2013). Importance of handicraft traditions in investigation of history of urban culture in Ganja. ISJ Theoretical \& Applied Science 11(7), 61-66. doi: http://dx.doi.org/10.15863/TAS.2013.11.7.10

The dawn of Art (1974). Leningrad: Aurora Art Publishers. 